\title{
TEACHING SITUATED DESIGN METHODS: A CASE STUDY
}

\author{
Sarah Lugthart, \& Michel van Dartel \\ Centre of Applied Research for Art, Design and Technology, Avans University of Applied Sciences \\ (Netherlands)
}

\begin{abstract}
Preparing students in higher vocational education for a career in the domain of design requires the transfer of a deep understanding of the design methods applied within that domain. Within design practices, however, a shift can be observed from methods based on sequences of planned steps towards the design of a certain object, to more situated design methods that favor progression on the basis of actions in the design context towards more open ended outcomes. Moreover, literature on situated design methods suggests that adopting such methods is to perform situated actions on the basis of previous experience, rather than to execute preplanned steps explained by educators. This poses a challenge to design educators, as students do typically not have much previous design experiences to rely on; they have yet to build up a reservoir of resources and know-how to be able to perform within a design context. To explore this apparent dilemma, we formulated the following research question: How can education best facilitate situated actions in the design processes of students? To gain insight into this question, a case study was conducted in which 25 students were observed and interviewed at various stages of their design processes in the context of a minor course on the design of mixed reality projects. Their processes were observed on the basis of natural observation, and data was recorded on the basis of written event sampling. At the end of each workshop, semi-structured interviews were conducted with participants about their design process. The case study revealed that (i) the discussion of story concepts in the classroom environment was dominant in the design actions taken by the students; (ii) planned and situated design actions generally alternated within their design processes; and (iii) limitations in time, facilities and pre-existing experience seemed important factors with respect to their choices in design actions. On the basis of these findings, we conclude that, design education should ultimately facilitate situated actions in the design processes of students by opening up the learning environment for situated learning experiences rather than merely use it for reflection on the outcomes of design steps. This conclusion raises fundamental questions about the organization of higher vocational education in design and calls for a better awareness of the relationship between situated design methods and the learning environments in which they are taught.
\end{abstract}

Keywords: Design education, situated action, design methods.

\section{Introduction}

Preparing students in higher vocational education for a career in the domain of design requires the transfer of a deep understanding of the practices within that domain (Cross, 2006; Goodman, Stolterman, \& Wakkary, 2011; Van Dartel, 2016). In literature on design practices that is used in support of this transfer, design processes are generally described as sequences of planned steps towards creating a certain design object (Simonsen et al., 2014). In contrast, an approach to design referred to as situated design methods favors progression on the basis of 'situated actions' in the design context (Simonsen et al., 2014). The concept of situated design originated in the work of Lucy Suchman, who conducted research on human-computer interaction that led her to a distinction between 'plans', i.e. processes that are dependent on predefined actions, and those that are based on 'situated actions', i.e., that "depend in essential ways upon the action's circumstances" (Suchman, 1987, p. 38). In contrast to planned steps, which are generally determined on the basis of preconceived ends, situated actions are informed by the specific circumstances of a design context (Simonsen et al., 2014). Consequently, in adopting situated design methods, designers generally rely on their experiences with particular material and social circumstances, rather than on explicit knowledge of design. Moreover, literature on situated design methods suggests that adopting such methods is to perform situated actions on the basis of previous experience, rather than to execute preplanned steps explained by educators (Simonsen et al., 2014). This poses a challenge to design educators, as students do typically not have much previous design experiences 
to rely on; they have yet to build up a 'grab bag of technical tricks', as Rouse and Barba (2017) call it. To explore this apparent dilemma, we formulated the following research question: How can education best facilitate situated actions in the design processes of students?

Besides its practical relevance for educators that aim to prepare students for a career in design, addressing this research question may also shed new light on two more fundamental challenges in design education. Firstly, the challenge that the increase in attention for practice-led education causes the borders between practice, research and education to be more and more permeable (Dunin-Woyset \& Nilsson, 2007). Secondly, a better understanding of how education should facilitate situated actions in the design processes of students may help to support educational institutions in resisting the push towards (over-)specifying the learning outcomes (Davies, 2012).

To address our research question, in the below, we introduce a case study in which we closely observe the design processes of students in higher vocational education and interview them about their design processes. Subsequently, we will discuss the results that our case study yielded and conclude on our research question.

\section{Case study: Minor research in immersive storytelling}

Recently, Rouse and Barba (2017), looked at processes in the design of mixed reality applications and concluded that agile approaches like prototyping seem to fall somewhere on a spectrum between opportunistic and deterministic. The design approaches on the opportunistic side of this spectrum typically leave room for serendipity and improvisation and are often taken by designers with an arts and humanities background. In line with what Suchman's calls 'situated action', Rouse and Barba conclude that designers with such backgrounds are more "willing to adapt to emergent phenomena" (2017, p. 250). Former experiences provide them with a "grab bag of technical tricks" (ibid.). On the other end of the spectrum, deterministic approaches are those in which designers tend to plan ahead, or what Suchman calls 'plans'. These approaches are significantly more often adopted by designers with interaction and media design backgrounds (Rouse \& Barba, 2017).

Moreover, Rouse and Barba's work on design approaches suggests that the domain of mixed reality is a fruitful domain for an exploration on how education can best facilitate situated actions in the design processes of students. Therefore, in our case study, students developed designs for mixed reality applications over the course of three workshops. These workshops were part of a minor program, titled Research in Immersive Storytelling, which taught students how to develop an immersive story and engage an audience in a story through some kind of mixed reality interaction. Each workshop ran for a period of two to three weeks and focused on a different theme: Prototype It (workshop 1) focused on the use of prototypes in interaction design; Hoax Design (workshop 2) focused on the design of transmedia projects; and $V R \&$ Performativity (workshop 3) focused on the design of Virtual Reality experiences. While students worked on individual projects in workshop 1, they collaborated on projects in groups of three or four students during workshops 2 and 3. Each workshop was set up according to the five stages of design thinking: (1) empathize, (2) define, (3) ideate, (4) prototype, and (5) test (Dam \& Siang, 2018). For the purpose of our case study, we observed students throughout all five stages of their design processes and subsequently conducted interviews with them about their design processes.

\subsection{Participants}

The students that participated in the case study majored in a variety of domains related to design and immersive storytelling, such as photography/film (PHOFI, 1 student), illustration/animation (ILAN, 9 students), fine art (FA, 2 students) and multimedia design (MD, 10 students). All 25 students (participants) had recently entered their third year of a four-year Bachelor's degree program of the Academy for Art and Design or of the Academy for Communication and User Experience of AVANS University of Applied Sciences (Breda, NL), with the exception of three participants that studied similar degree programs at Fontys Media Design (Tilburg, NL), Luca School of Arts (Brussels, BE) and Llubjana Academy of Art \& Design (Llubjana, SLO). All 25 students participated in each of the three workshops of the minor program.

\subsection{Observations and interview}

During each workshop, participants worked in various classrooms that were designated to the minor program at predetermined hours, except for workshop 3, in which one classroom was dedicated to the workshop during school opening hours. During these hours, the design processes of participants were observed on the basis of natural observation. These observations were recorded on the basis of written event sampling; whenever design steps were evaluated amongst participants, e.g., when methodology was explicitly discussed or a certain type of planning took place, their discussions would be captured in writing (Lewis-Beck, Bryman, \& Futing Liao, 2004). 
At the end of each workshop, a sample of five participants were selected for semi-structured ten-minute interviews about their design processes. Participant samples always included participants from at least two different academies and included both male and female students. In each interview, participants were first asked to describe the steps they took in their design processes to achieve their results. Secondly, participants were invited to elaborate on their choices for these particular steps. Thirdly, interviewees were asked to explain their design approach and whether this approach was based on either pre-determined plans (i.e. deterministic approach) or situated actions (i.e. opportunistic approach). Students were introduced to these two design approaches during the introduction to the minor program, but were not explicitly asked to apply them within the workshops. Audio recordings of each interview were made, which were subsequently transcribed.

\section{Results}

Our case study yielded event samples related to each of the five stages of design thinking that the workshops were structured around, as well as fifteen interviews (five per workshop) that reflected on the design processes of participants.

\subsection{Observations}

The written event samples that our case study yielded show that, within the empathize, define and ideate phases of the design processes observed, design actions were dominated by brainstorms and discussions of story concepts: Participants typically gathered around tables to discuss a concept with their teacher(s) and other students that were present. In some occasions, sketches were used to illustrate the concept, but in the majority of observations the exchanges were purely verbal. The only exception to this occurred during workshop 3, which involved experimentation with a $360^{\circ}$ camera in small groups outside of the classroom environment followed by the design of experiences using this technology within the classroom.

Each workshop ended with a presentation of outcomes, which were considered to embody the testing phase of design thinking, since these presentations were a way for students to test their concepts and reflect on them in the presence of teachers and fellow students. With the exception of workshop 3, the realization of prototypes, part of the prototype phase, did typically not take place within the classroom environment during workshop hours, but was considered homework and therefore typically conducted outside of it.

In the light of our research question, the seemingly most notable observation that our case study brought forward is that, design actions within the classroom environment seem to concentrate on brainstorming and discussing the story concept.

\subsection{Interviews}

Overall, the interviews conducted with participants that our case study yielded revealed that students are able to distinguish multiple steps in their design processes. Participants often diverge into the aims of their projects and explaining their concepts, but a small reminder would be enough to regain focus on the design steps taken, after which they would distinguish between steps such as brainstorming, visualizing, prototyping and testing.

In explaining why certain steps were taken in their design processes, students typically mentioned reasons that related to either time constraints or to limits imposed by the workshop facilities. Students for instance mentioned that "My initial idea was really big in my head, but because of time constraints I had to make it much smaller and concrete." (workshop 1, Brussels); "It would probably have helped a lot if I had seen the space first and would have started working from there. That is just how I work, but this was not possible in this case." (workshop 1, Llubjana); "I had a hard time to not be able to explore as much as I wanted. This made me insecure about my decisions." (workshop 2, FA); "Within a day we came up with our concept and we almost immediately started creating a space using materials that we could easily get our hands on." (workshop 3, MD); and "Then I ran into what I usually run into: 'yeah nice plan, but I'll never be able to realize it in the short time that is available.." (workshop 3, ILAN).

Asked to indicate whether their design steps were based on pre-determined plans (i.e. deterministic approach) or situated actions (i.e. opportunistic approach), participants would typically answer that their design approach were based on determinism as well as opportunism, and that they had been alternating between these approaches at different stages of their design processes. Participants would for instance respond with phrases such as: "I was a bit done with thinking about the concept, so I just starting making." (workshop 1, FA); "I might have a plan, but that plan does not work out half of the time, which is when I turn to just responding on the fly to whatever happens while making. But before that, when I'm thinking about what kind of support I need from people, [...] I base my actions on plans." (workshop 1, MD); "When I sit down to focus on my idea, it doesn't work. I just have to continue with my life and then ideas start flowing automatically. When I am riding my bike for instance." (workshop 1, 
$\mathrm{MD}$ ); and "In the beginning we had a plan, but then when we started to make we relied more on improvisation." (workshop 3, MD). In some cases, participants also reflected on their reliance on pre-existing knowledge. They would for instance add comments like "I know what my technical limitations are, so I knew I could do this myself." (workshop 1, MD); "We planned it all in detail beforehand, but only knew whether or not it was going to work when we executed it." (workshop 2, ILAN); and "We were being modest in formulating initial ideas, because we thought they would otherwise be impossible to realize, but when we tried our ideas out using VR it was all much easier than we thought." (workshop 3, MD).

In the light of our research question, the most notable insights that the interviews with students about their design processes provided us with were that students alternated planned and improvised actions within their design process and that time, facilities and previous experience (e.g., what participants assumed to be feasible) are important factors with respect to the choices that students make in their design processes.

\section{Discussion}

While the observations of the design processes of participants (3.1) gave rise to the noteworthy insight that (i) within the classroom environment, design actions seem to concentrate on brainstorming and discussing the story concept, from the interviews conducted with participants in the case study (3.2) it can be gathered that (ii) planned and improvised actions alternated within the design process, as well as that (iii) time, facilities and previous experience are important factors with respect to the choices that students make in their design processes. In the below, we will discuss these three findings in more depth.

\subsection{The classroom environment}

Our findings suggest that design methods based on plans dominate in the classroom environment. Although this could imply a causal relationship between the design actions conducted and the classroom environment, a mere practical explanation is also possible: During the first half of the minor program students met in different classrooms, while they were provided with a dedicated classroom to work in only during the second half of the minor. This may have had an influence on the nature of the actions undertaken in the classroom in the earlier stages of design, since initially classrooms were set up to meet and discuss, instead of for instance work on prototyping designs. In contrast, in the second half, during workshop 3 participants expressed that they had started making within the context of the school environment relatively soon after the initial brainstorm, since that was also part of an assignment. It seems that the classroom or school environment limited the possibilities for developing concepts by way of situated actions, in the case of workshop 1, or helped to facilitate it, in case of workshop 3, depending on how the class or school environment was used. In short, when the classroom environment did not provide the facilities required to perform design actions beyond the creation and discussion of plans, participants took such actions outside of this environment (see, e.g., the quote "I was a bit done with thinking about the concept, so I just starting making." reported in Subsection 3.2). This finding simultaneously marks the limits of natural observation in the classroom environment as a means to analyze design processes: situated actions were typically conducted outside of the classroom. Most of these actions undertaken by participants in our case study therefore only became apparent in the interviews conducted.

\subsection{Plans and situated actions}

Our results suggest that, for most participants, the concept phase of a design process is characterized by a planned design approach. However, as one participant explained, such ideation often leads to follow up outside of classroom environment, which might be more improvisational in nature. This makes it difficult to strictly discern planning from improvising in the design process. If anything, our results suggest that design students moreover alternate between the two types of approaches. This finding seems to bring nuance to the argument brought forward by Rouse and Barba (2017) that designers can be positioned somewhere on the spectrum between opportunistic or deterministic. In line with Suchman (1987), our findings confirm that, at least for the participants in our case study, plans become a resource in situated design, rather than predetermining the course of action in any strong sense, as Simonsen et al. (2014) claim. Therefore, it is important to note that students alternate between these approaches, disregarding whether this alternation is for instance caused by the format of a workshop or the facilities available.

\subsection{Issues of time, facilities and previous experience}

Based on the insight that time, facilities and previous experience are important factors with respect to the choices that students make in their design processes, it could be argued that the context in which the design should function or be experienced is ultimately the best place to design it. The results 
yielded during workshop 2 illustrate this, as participants reported that they could only find out whether or not their hoax-concepts would 'work' if they executed it. Clearly, in this case, the classroom environment provided limitations that the design context did not.

Finally, our findings also suggest that participants often relied on ways of working that they had previous experience with, as many quotes from the interviews start with phrases such as "I'm the type of designer that ..." or "I'm someone that ...". Moreover, our findings reveal that when certain expertise was required to realize a concept but was lacking in a participant's 'grab bag of technical tricks', students often resort to improvisation to work around this lack in experience. As reported above, one student for instance mentioned that "I might have a plan, but that plan does not work out half of the time, which is when I turn to just responding on the fly to whatever happens while making." On the basis of such improvisation students may gain experiences that they add to their 'grab bag of technical tricks', instead of limiting themselves to what they already know.

\section{Conclusions and future research}

Our case study gave rise to three important insights in the light of our research question How can education best facilitate situated actions in the design processes of students? Firstly, design actions seem to revolve around brainstorming and discussing within the classroom environment. Secondly, planned and improvised actions alternated within the design processes of students. And thirdly, time, facilities and previous experience are important factors with respect to choices in the design actions taken by students.

On the basis of these insights, we conclude that design education should ultimately facilitate situated actions in the design processes of students by opening up the learning environment for situated learning experiences rather than merely use it for reflection on the outcomes of design steps. By doing so, educators can guide students in the improvisation required to gain experiences and expand their 'grab bag of technical tricks', which may be alternated with preplanned steps. Future research should look into how the education environment can best be opened up for situated learning experiences. As workshop 3 in our case study seemed to facilitate situated action best, it might particularly be worth looking in detail at lab-like environments for the facilitation of situated actions in the design processes of students.

Besides its practical relevance for educators that aim to prepare students for a career in design, our conclusion also sheds new light on two more fundamental challenges in design education: Firstly, better facilitation of situated action may be important in the move towards more practice-led education, which causes the borders between practice, research and education to be more and more permeable (Dunin-Woyset \& Nilsson, 2007). Secondly, a better understanding of the role and relevance of situated actions in the design processes of students may help to resist the (over-)specifying of learning outcomes (Davies, 2012) by offering alternative ways of looking at the outcomes of design processes.

\section{References}

Cross, N. (2006). Designerly Ways of Knowing. Basel: Birkhäuser.

Dam, R. \& Siang, T. (2018, March 31). 5 Stages in the Design Thinking Process. Retrieved from https://www.interaction-design.org/literature/article/5-stages-in-the-design-thinking-process.

Van Dartel, M.F. (2016). Aesthetics in the Wild: Art and Design Practices and Pedagogies after the Situated Turn. Breda, NL: Avans.

Davies, A. (2012, July). Learning Outcomes and Assessment Criteria in Art and Design. What's the recurring problem? Networks, $18 . \quad$ Retrieved http://arts.brighton.ac.uk/projects/networks/issue-18-july-2012/learning-outcomes-and-assessmentcriteria-in-art-and-design.-whats-the-recurring-problem

Dunin-Woyset, H. \& Nilsson, F. (2007). Design Education, Practice, and Research: on building an inquiry. Studies in Material Thinking. Volume 11, 3-17.

Goodman, E., Stolterman, E., \& Wakkary, R. (2011). Understanding Interaction Design Practices. Proceedings of the SIGCHI Conference on Human Factors in Computing Systems, 1061-1070.

Lewis-Beck, M.S., Bryman, A., \& Futing Liao, T. (2004). The SAGE Encyclopedia of Social Science Research Methods. Thousand Oaks, CA: Sage Publications.

Rouse, R. \& Barba, E. (2017). Design for Emerging Media: How MR Designers Think about Storytelling, Process, and Defining the Field. N. Nunes, I. Oakley, and V. Nisi, (eds.). Proceedings ICIDS2017 International Conference on Interactive Digital Storytelling, 245-258.

Simonsen, J., Svabo, C., Strandvad, S., Samson, K., Hertzum, M. \& Hansen, O. (eds.). (2014). Situated Design Methods. Cambridge, MA: MIT Press.

Suchman, L. (1987). Plans and Situated Actions: The Problem of Human-Machine Communication. New York, NY: Cambridge University Press. 